\title{
Dentistry's role in the diagnosis and co-management of patients with sleep apnoea/hypopnoea syndrome
}

\author{
A. H. Friedlander, ${ }^{1}$ I. K. Friedlander, ${ }^{2}$ and M. A. Pogrel, ${ }^{3}$
}

The sleep apnoea/hypopnoea syndrome (SAHS) is characterized by repeated upper airway narrowing or collapse during sleep. The obstruction is caused by the soft palate and/or base of tongue collapsing against the pharyngeal walls because of decreased muscle tone. These episodes are accompanied by hypoxaemia, surges in blood pressure, brief arousal from sleep and pronounced snoring. Individuals with occult disease are at heightened risk of motorway accidents because of excessive sleepiness, sustained hypertension, myocardial infarction, and stroke. The signs and symptoms of SAHS may be recognisable in the dental practice. Common findings in the medical history include daytime sleepiness, snoring, hypertension, and type 2 diabetes mellitus. Common clinical findings include male gender, obesity, increased neck circumference, excessive fat deposition in the palate, tongue (macroglossia) and pharynx, a long soft palate, a small recessive mandible and maxilla, and calcified carotid artery atheromas on panoramic and lateral cephalometric radiographs. Dentists who recognise these signs and symptoms have an opportunity to diagnose patients with occult SAHS. After confirmation of the diagnosis by a physician, dentists can participate in the management of the disorder by fabricating mandibular advancement appliances that enlarge the retroglossal space by anterior displacement of the tongue and performing corrective upper airway surgery that prevents recurrent airway obstruction.

${ }^{1}$ Associate Chief of Staff for Graduate Medical

Education, VA Greater Los Angeles Healthcare

System, Director of Quality Assurance, Hospital

Dental Service, UCLA Medical Center, and Professor,

Oral and Maxillofacial Surgery, UCLA School of

Dentistry, Los Angeles, CA; ${ }^{2}$ Public Health Nurse at

the West Valley/Los Angeles County Department of

Health in Panorama City, California and a Research

Associate at the VA Sepulveda Dental Service,

Sepulveda, CA; ${ }^{3}$ Professor and Chairman,

Department of Oral and Maxillofacial Surgery,

University of California San Francisco School of

Dentistry, San Francisco, $C A$

Correspondence to: A. H. Friedlander, Associate Chief

of Staff for Graduate Medical Education, VA Greater

Los Angeles Healthcare System, 11301 Wilshire Blvd,

Los Angeles, CA 90073, USA

e-mail address: arthur.friedlander@med.va.gov

ReFEREED PAPER

Received 20.12.00; Accepted 15.3.00

(C) British Dental Journal 2000; 189: 76-80
In brief

- Preliminary diagnosis of patients with occult SAHS.

- Formulation of a sophisticated consultation request to a sleep specialist for confirmatory diagnosis.

- Medical diagnostic and treatment modalities used to manage patients with SAHS.

- Dental and oral surgical treatments available to manage patients with SAHS and their role in the comprehensive care plan.
Charles Dickens, an acute observer of the human condition, vividly described the clinical features of sleep apnoea/hypopnoea syndrome (SAHS) when he characterized the 'fat boy' in the Pickwick Papers: 'Joe was obese, excessively sleepy, snored loudly and suffered from heart failure.' ${ }^{1}$ SAHS is typified by the periodic narrowing of the pharyngeal airway during sleep and results in absent (apnoea) or diminished (hypopnoea) airflow into the lungs despite persistent inspiratory effort. These obstructive episodes which may occur at various levels from the nasopharynx to the base of tongue and the hypopharynx lead to hypoxaemia, increased ventilatory effort, brief arousal from sleep, daytime somnolence and cardiovascular disease. SAHS occurs most commonly during rapid eye movement (REM) sleep when the dilator muscles of the upper airway (geniohyoid, genioglossus, tensor veli palatini) lose their tone and are unable to offset the negative pressure of inspiration which draws the base of tongue (including the epiglottis) and soft palate posteriorly against the pharyngeal walls (Figs 1 and 2). ${ }^{2}$ The purpose of this paper is to alert dentists that in the course of performing a comprehensive examination, they may identify patients with occult disease, participate in their care, and possibly save lives.

Epidemiological studies in the United Kingdom estimate that $5.7 \%$ of men and $1.2 \%$ of women in the 35-69 year age group have SAHS. ${ }^{3}$ In Britain, daytime sleepiness associated with SAHS is linked to increased use of medical care. ${ }^{4}$ Impaired vigilance and driving performance have also been recognised by the Leicestershire police study which has noted that around $20 \%$ of accidents on motorways in the county are caused by motorists falling asleep. ${ }^{5}$ Thus, clinicians in the UK are required to advise patients with SAHS that they should not drive until effective treatment has been established. ${ }^{6}$ Likewise, studies in Europe have shown that SAHS associated daytime sleepiness leads to motorway and work 


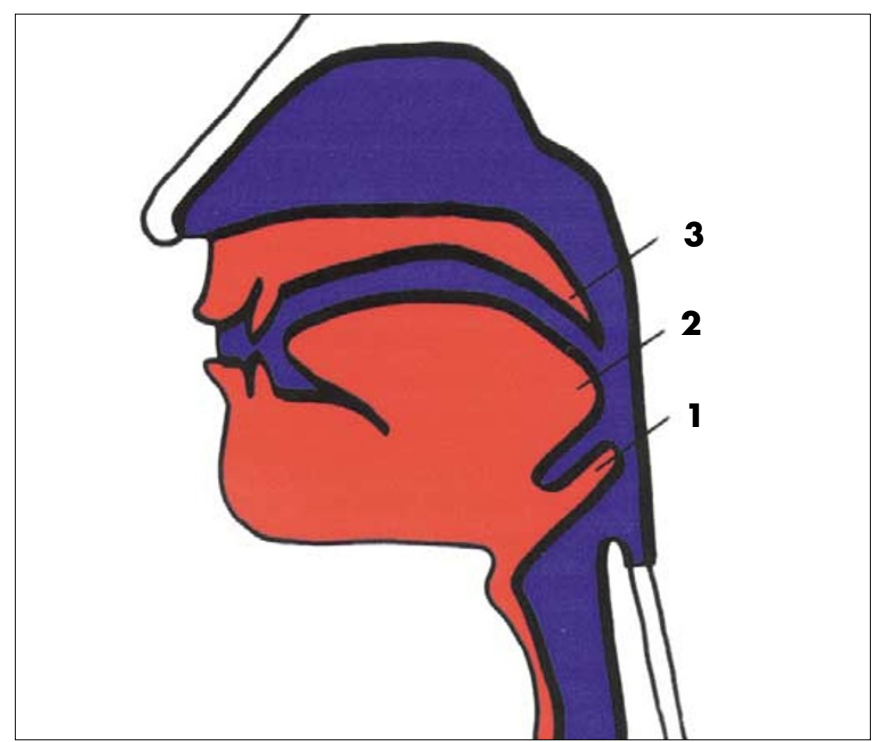

Fig. 1 Line drawing illustrating the normal (unobstructed) anatomy of the upper airway when sleeping in the supine (on back) position. Note that the epiglottis (1), base of tongue (2), and soft palate (3) are distant from the posterior pharyngeal wall

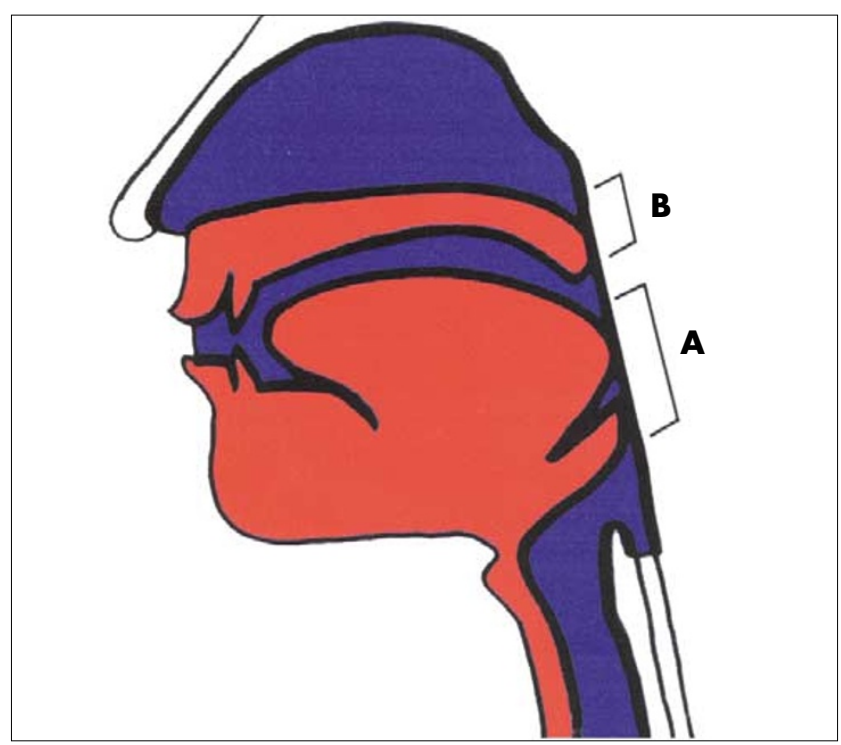

Fig. 2 line drawing illustrating the obstructed airway of an individual with SAHS when sleeping in the supine position. Note that the epiglottis and tongue (A), and soft palate (B) are collapsed against the posterior pharyngeal wall and block the flow of air related accidents costing nation/states the equivalent of billions of pounds-sterling each year in medical care costs and lost productivity. $^{7-9}$

\section{Pathophysiology}

The periods of compromised airflow usually last 10-30 seconds and result in repetitive asphyxia [fall in blood oxygen (hypoxaemia) and a rise in blood carbon dioxide (hypercarbia)], transient hypertension and bradycardia (a heart rate of less than 60). The surges in systolic blood pressure $(20-40 \mathrm{~mm} \mathrm{Hg}$ ) are precipitated by a hypoxaemia induced rise in catecholamine production and sympathetic nervous output which cause constriction in vascular smooth muscle. ${ }^{10}$ Also responsible for the transient hypertension is the increased (though frustrated) respiratory effort. ${ }^{11}$ The bradycardia is also caused by the hypoxaemia and results from carotid body stimulation of the vagus nerve. ${ }^{12}$

Powerful efforts to inspire against the closed upper airway continues but breathing resumes only when the subject transiently awakens (sleep fragmentation); often without recall. This arousal restores the tone in the muscles surrounding the pharynx, allowing the individual to gasp and then breathe without obstruction. Upon opening of the airway, cardiovascular and pulmonary derangements are rectified. Even when the airway is open, partial obstruction frequently occurs and results in loud, irregular snoring sounds caused by air rushing through the narrow passage and stimulating the soft palate, uvula, throat walls and tongue to vibrate. However, once asleep again, the obstruction rapidly recurs, and the cycle repeats hundreds of times a night.

The recurrent sleep disruption causes troublesome (and often disabling) daytime sleepiness, fatigue, morning headache, diminished libido, impotence, decrements in cognitive performance (impaired memory and concentration), mood disorders (episodes of irritability and depression), and poor performance in the work place. Recurrent exposure to apnoeic initiated hypoxaemia, hypercarbia, and increased catecholamine production and sympathetic activity with resultant surges in systolic blood pressure are known to be associated with: sustained hypertension, coronary artery atherosclerosis and ischaemic stroke. ${ }^{13-16}$ The atherosclerotic process is accelerated in patients with SAHS because of the combined effects of hypertension, hypoxaemia, and increased sympathetic activity. ${ }^{17}$ Our research group has recently observed that around one-third of patients with SAHS also have type 2 diabetes mellitus which further accelerates the process of atherosclerosis. ${ }^{18}$

Almost all individuals with SAHS snore, however, not all individuals who snore have SAHS. In fact, the majority of individuals who make snoring sounds while sleeping do so because their uvula and/or posterior border of soft palate is excessively lax and vibrates as air passes over it during normal respiration. Snoring sounds whether associated with SAHS or of a benign nature may be so pronounced that the bed mate is unable to sleep with the individual and the noise is cited as a reason for divorce. ${ }^{19}$

\section{Medical diagnostics}

A clinician may perform an awake video nasopharyngoscopy under local anaesthesia and ask the patient to perform a reverse-Valsalva manoeuvre (ie take a deep inspiration with a closed nose and mouth) in order to locate the site of airway collapse (base of tongue, pharyngeal walls or soft palate). Respiratory physicians confirm the diagnosis of SAHS by polysomnography, an overnight sleep study conducted in a laboratory with computerised recording equipment and the presence of technical staff. Neurophysiologic tests determine the sleep stage and arousals [electroencephalography/ EEG, electromyography/EMG (submental muscle tone) and electro-oculography (eye movements)], respiratory tests measure oxygen intake [oronasal airflow, rib cage and abdominal wall motion, arterial oxyhaemoglobin saturation)], a throat microphone detects snoring sounds and cardiological tests document secondary cardiovascular changes (electrocardiograph/ ECG and blood pressure)]. Recently, some institutions have begun to document SAHS by use of a portable sleep monitoring device in the patient's home. ${ }^{20}$

The apnoea/hypopnoea index (AHI) [also termed the respiratory disturbance index (RDI)] is used by many clinicians to confirm the diagnosis and to quantify the severity of illness. The AHI is calculated by adding the number of apnoeas (cessation of breathing for 10 seconds or more) and hypopnoeas [reduction in tidal volume accompanied by a $3 \%$ or greater fall in oxy- 
PRACTICE $\underline{\text { medical matters }}$

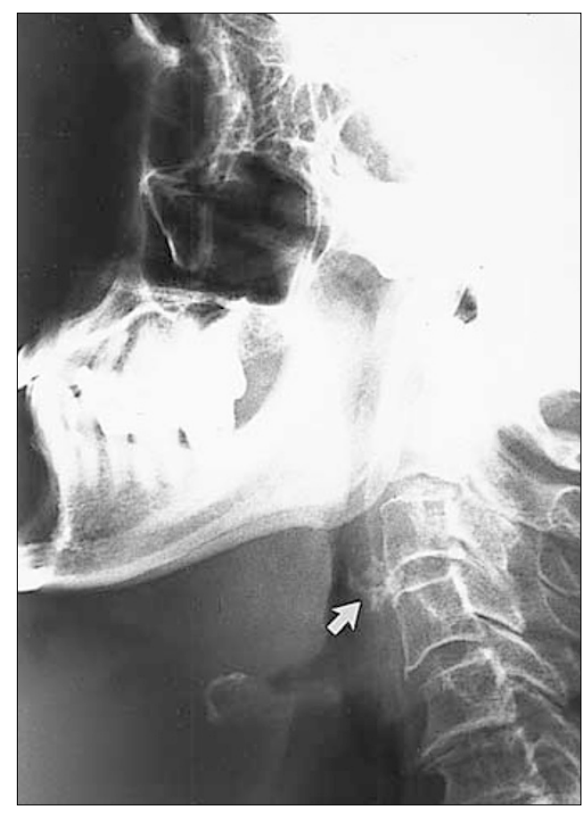

Fig. 3 Cephalometric radiograph showing a diffusely calcified carotid artery atheroma (arrow)

gen saturation $\left(\mathrm{S}_{\mathrm{a}} \mathrm{O}_{2}\right)$ or arousal from sleep] for the duration of the study and dividing that by the number of hours slept. Mild SAHS is defined as an AHI of 5-20 per hour of sleep, moderate SAHS as an AHI of 20-40 per hour of sleep and severe SAHS as an AHI greater than $40 .^{21}$

\section{Dental diagnostics}

A dentist may be the first healthcare provider to identify an individual with SAHS because its salient signs and symptoms are often recognisable in the dental practice. Common findings in the medical history include: age over 45 , male gender, daytime sleepiness, snoring, hypertension, difficult tracheal intubation for the administration of a general anesthetic, and type 2 diabetes mellitus. ${ }^{22}$ Common clinical findings include: mild-moderate obesity, [a body mass index (BMI, measured in weight $/$ height $^{2}$ ) greater than $29 \mathrm{~kg} / \mathrm{m}^{2}$ ] and a neck circumference greater than $42.5 \mathrm{~cm}$ (subcutaneous masses externally compress the pharynx), excess fat deposition in the palate and tongue (possibly causing a true macroglossia) and pharynx (intrapharyngeal/visceral fat masses narrow the airway). ${ }^{23}$ Also at risk are individuals with small (micrognathia) and repositioned (retrognathia) jaws. The tongue, be it enlarged or of normal size but with reduced functional space (relative macroglossia) is forced posteriorly toward the pharyngeal wall and superiorly above the plane of occlusion. Likewise, the soft palate (which is usually elongated and enlarged) is positioned posteriorly, adjacent to the pharyngeal wall. The uvula is not usually seen during phonation because it lies below the base of the posteriorly/superiorly positioned tongue. $^{24}$

On occasion, dentists are consulted by physicians and asked to obtain and evaluate lateral cephalometric radiographs of patients with suspected SAHS. The radiographs of individuals with SAHS usually confirm a retropositioned maxilla and mandible, a shortened cranial base, an inferiorly set hyoid bone, enlarged soft palate and tongue, and a narrowed posterior airway space. ${ }^{25}$ Our research group has recently observed that approximately onefifth of these radiographs also reveal calcified carotid artery atheromas (Fig. 3). ${ }^{26}$

\section{Medical management}

In the early to mid-1980s sleep clinics in the UK began to treat substantial numbers of patients. The main indication for treatment then and now remains subjective daytime sleepiness. ${ }^{27}$ Physicians in these clinics initially prescribe weight reduction because it is often associated with a loss of fat surrounding the airway thereby allowing it to dilate more fully. Unfortunately, permanent weight loss for these individuals is even more difficult than it is for the rest of the populous because they have a lowered basal metabolic rate. Other behavioral interventions include the cessation of habits that tend to worsen apnoea [(ie inadequate sleep, smoking, consumption of ethanol (relaxes walls of upper airway permitting collapse), and use of benzodiazepines, narcotics, barbiturates)]. ${ }^{28}$

If these remedies fail, the physician will then likely prescribe the administration of continuous positive airway pressure. ${ }^{29}$ More than 2000 such prescriptions were given in the UK in $1995 .{ }^{30,31}$ Known as CPAP, a low pressure blower delivers a continuous stream of air into a sealed nasal mask that the patient wears while sleeping. The positive pressure pneumatically splints the airway open by preventing the soft palate and tongue from collapsing against the pharyngeal walls while permitting periodic expiration. CPAP corrects arterial oxyhaemoglobin desaturation, eliminates carbon dioxide retention, restores the normal drive to breathe, stabilizes blood pressure and permits continuous unfragmented sleep. CPAP also reduces patients' sleepiness and improves their cognitive performance and mood. The Oxford Healthy Life Survey conclusively showed a marked improvement in the self-reported physical, mental, and social domains of individuals with SAHS living in Berkshire, Buckinghamshire, Northhamptonshire and Oxfordshire counties who were afforded CPAP therapy. ${ }^{32}$ The findings of the Australian National Health Medical Research Council are congruent with those of the Oxford survey and in addition they show that CPAP is highly cost effective. ${ }^{33}$ The report notes that individuals who successfully use CPAP gain an average of 5.5 quality adjusted life years (QALYs) at an approximate cost of

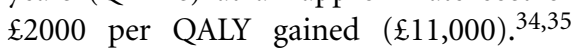
Unfortunately, only two out of three patients use the device long-term and many use it only for limited hours during sleep. ${ }^{36}$ Individuals with a class two malocclusion are almost uniformly uncomfortable with the fit of the CPAP mask and often do not use it as prescribed. Patient compliance is also poor because of dryness, burning, and congestion of nasal mucosa, machine noise, and less intimacy with bed partner. ${ }^{37}$

\section{Dental and surgical management}

The Royal College of Physicians of England in its most recent publication on the subject of SAHS has acknowledged the participatory role of dentists in managing patients with the disorder. The College noted that there is increasing evidence that intra-oral devices that advance the mandible during sleep and thus prevent the tongue and soft palate from collapsing against the pharyngeal wall are useful in the management of patients with mild to moderate SAHS. ${ }^{38}$ The RCP based its recommendation on the results of studies which have shown that these devices improve overnight breathing patterns and symptoms. ${ }^{39}$ Bolstering these results are additional studies which have shown that the oral appliances decrease the AHI by more than half and improve both arterial oxygen saturation and sleep quality in approximately $50 \%$ of patients with SAHS. ${ }^{40-42}$

Removable mandibular repositioning devices are constructed so that the lower jaw 


\section{PRACTICE $\underline{\text { medical matters }}$}

is positioned several (2-5) millimetres anteriorly (between $50 \%$ and $100 \%$ of the patient's maximum protrusive distance), thereby advancing the tongue passively because of its attachment to the genial tubercles. ${ }^{43-45}$ These devices simultaneously move the soft palate anteriorly because of its attachment to the tongue via the palatoglossus muscle. ${ }^{46}$ These movements enlarge the airway and reduce the likelihood that the tongue or soft palate will collapse against the posterior pharyngeal wall when the patient inspires during sleep. These devices can be fabricated as either one or two piece appliances. A fixed/one-piece appliance positions the mandible anterior to the maxilla and is retained in place by the use of clasps, acrylic or thermoplastic polymer (Fig. 4). Anterior breathing holes can be developed in the appliance so that oral respiration is permitted for those with restricted nasal air flow. A non-fixed/two piece (a separate appliance for each arch) device positions the mandible anteriorly and is secured to the maxilla by use of thermoplastic buttons, inter-arch elastics or a buccal tube and rod. The twopiece appliance affords the patient greater comfort by permitting anterior and lateral mandibular movement (Fig. 5). Adverse side effects associated with both the one piece and two piece mandibular advancement appliances include the encouragement of caries, gingivitis, excessive salivation, a temporary ( 15 to 30 minutes) bite change each morning and transitory tooth, jaw and temporomandibular joint tenderness. Side effects are usually mild and improve with time. There are major design differences in the numerous devices that are available and these differences impact success and compliance use rates. Consistent with the team approach to care and because efficacy varies, patients using these appliances should have a follow up polysomnogram in order to assure therapeutic adequacy. ${ }^{47,48}$

Individuals with SAHS may require surgery if they are unable to comply with CPAP, tolerate a prosthetic device or if larger movements (6 to $12 \mathrm{~mm}$ ) of the tongue are necessary in order to move it anteriorly and away from the posterior pharyngeal wall. If the respiratory obstruction can be documented as occurring in the

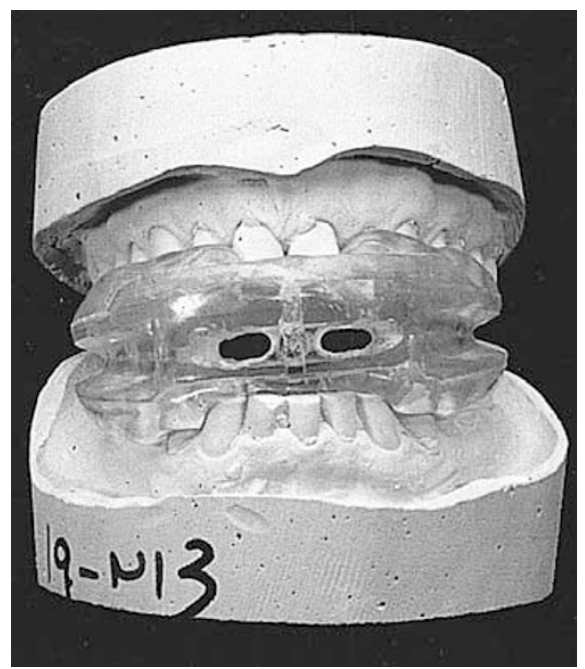

Fig. 4 Two-piece mandibular advancement appliance (frontal-view)

retropalatal area, the otolaryngologist or oral and maxillofacial surgeon will resect tissue from the free border of the soft palate (including the uvula), posterior tonsillar pillars, the palatine tonsils (if still present) and excessive mucosa from the anterior pillars and posterior pharyngeal walls using a scalpel and general anesthesia in a hospital operating room (uvulopalatopharyngoplasty: UPPP). A more modest resculpting of the soft palate can be performed using a surgical carbon dioxide laser (laser assisted uvulopalatoplasty: LAUP), with a special attachment to protect the posterior pharyngeal wall, local anesthesia and intravenous sedation in an ambulatory surgery setting. ${ }^{49,50}$ These procedures are associated with significant post-operative discomfort and may result in palatal incompetence with nasal regurgitation on swallowing and nasal speech.

If the respiratory obstruction is documented as occurring at the base of the tongue and the patient is retrognathic, an oral and maxillofacial surgeon will advance the entire mandibular alveolus forward and reposition it into a physiologic class one occlusion. The anterior movement of the alveolus is accompanied by anterior movement of the tongue (away from the posterior pharyngeal wall) because the tongue is attached to the lower jaw at the genial tubercles. The tongue can be drawn even further anteriorly by performing a genioplasty which encompasses the general tubercles. Positioning the chin forward advances the attached geniohyoid and genioglossus muscles thereby repositioning the hyoid bone and base of tongue anteriorly. ${ }^{50-53}$ To further secure the hyoid bone in this position some surgeons ligate it to the mandible with Mersilene sutures or facia lata.

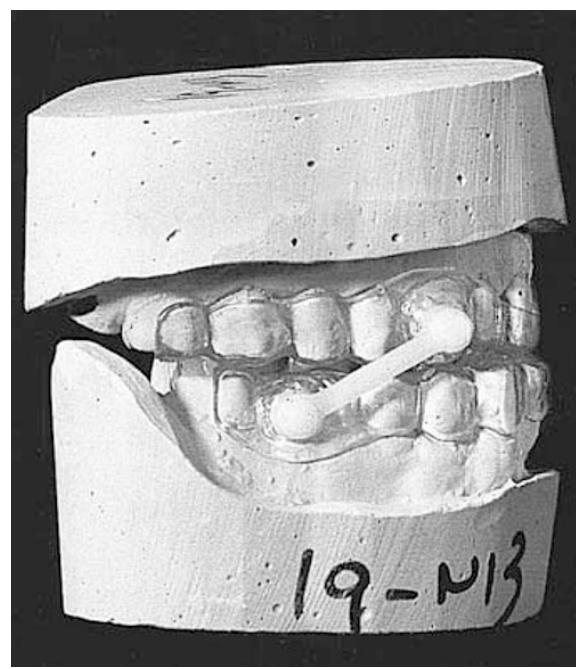

Fig. 5 Two-piece mandibular advancement appliance (lateral-view)

If the patient is not retrognathic, and the respiratory obstruction is documented as occurring at the base of tongue or at the base of tongue and soft palate, simultaneous surgical advancement of the maxilla and mandible is indicated. The maxilla is arbitrarily advanced $10-12 \mathrm{~mm}$ and the mandible is advanced an appropriate distance such as that the patient's class one occlusion remains intact. An advancement genioplasty can also be performed at this time if required. The results of this surgery while not always aesthetic do appear to obviate SAHS in most instances. ${ }^{54,55}$

Patients who have had soft palate and jaw surgery but who remain with severe symptomatic SAHS require a permanent tracheostomy to properly breathe at night. During the day however, the tracheal stoma may be obturated so as not to interfere with the activities of daily living. ${ }^{56}$

In conclusion, it must be emphasized that dentistry in concert with medicine has much to offer patients with SAHS. It is hoped that this presentation will alert general dentists to diagnose the patient with occult SAHS and join with physicians in offering this special group of patients the full range of available treatment options in order to defeat this potentially fatal illness.

The author gratefully acknowledges Ann Oliver, Dental Service Program Assistant for her assistance.

1 Cosnett J E. Charles Dickens: Observer of sleep and its disorders. Sleep 1992; 15: 264-267.

2 Douglas N J, Polo O. Pathogenesis of obstructive sleep apnoea/hypopnoea syndrome. Lancet 1994; 344: 653-655.

3 Davies R J, Stradling J R. The epidemiology of sleep apnoea. Thorax 1996; 51(Suppl 2): S65-S70.

4 Fleetham J A. A wake up call for sleep disordered breathing. BMJ 1997; 314: 339-340. 
5 Semple S J G, Gibson G J. Sleep apnoea and related conditions. J Royal Coll Physicians 1993; 27:363-364

6 Taylor J F. Medical aspects of fitness to drive: a guide for medical practioners. Medical Commission on Accident Prevention. London: HMSO, 1995

7 Haraldsson P O, Carenfelt C, Diderichsen F, Nygren A, Tingvall C. Clinical symptoms of sleep apnea syndrome and automobile accidents. ORL 1990; 52: 57-62.

8 Teran-Santos J, Jimenez-Gomez A, CorderoGuevara J. The association between sleep apnoea and the risk of traffic accidents. Cooperative Group Burgos-Santander. N Engl J Med 1999; 340: 847-851.

9 Ytterstad B, Norton R. Does CPAP prevent injuries? Lancet 1998; 351: 692.

10 Stradling J R. Epidemiology of snoring and its consequences. Monaldi Arch Chest Dis 1995; 50: $123-128$.

11 Stradling J R. Sleep apnoea and systemic hypertension. Thorax 1989; 44: 984-989.

12 Zwillich C W. Sleep apnoea and autonomic function. Thorax 1998; 53(Suppl 3): S20-S24.

13 Wilcox I, McNamara S G, Collins F L, Grunstein R R, Sullivan C E. 'Syndrome Z': the interaction of sleep apnoea, vascular risk factors and heart disease. Thorax 1998; 53(Suppl 3): S25-S28.

14 Sullivan C E, McNamara S G. Sleep apnoea and snoring: potential links with vascular disease. Thorax 1998; 53(Supp 3) S8-11.

15 Young T, Finn L. Epidemiological insights into the public health burden of sleep disordered breathing: sex differences in survival among sleep clinic patients. Thorax 1998; 53(Suppl 3 ) S16-S19.

16 Hoffstein V. Is snoring dangerous to your health? Sleep 1996; 19: 506-516.

17 Ferguson K A, Fleetham J A. Sleep-related breathing disorders: 4-consequences of sleep disordered breathing. Thorax 1995; 50: 998 1004

18 Friedlander A H, Friedlander I K, Yueh R, Littner M R. The prevalence of carotid atheromas seen on panoramic radiographs of patients with obstructive sleep apnoea and their relation to risk factors for atherosclerosis. J Oral Maxillofac Surg 1999; 57: 516-522.

19 Heath M. Management of obstructive sleep apnoea. Br J Nursing 1993; 2: 802-804.

20 Douglas N J. Respiratory dreams and nightmares. Br J Hosp Med 1997; 57: 95-98.

21 Pack A L. Obstructive sleep apnea. Adv Int Med 1994; 39: 517-567.

22 Hiremath A S, Hillman D R, James A L, Noffsinger W J, Platt P R, Singer S L. Relationship between difficult tracheal intubation and obstructive sleep apnoea. $\mathrm{Br} \mathrm{J}$ Anesth 1998; 80: 606-611.

23 Hoffstein V, Mateika S. Differences in abdominal and neck circumferences in patients with and without obstructive sleep apnoea. Eur Resp J 1992; 5: 377-381.

24 Waite P D. Obstructive sleep apnoea: a review of the pathophysiology and surgical management. Oral Surg Oral Med Oral Path
Oral Radiol Endo 1998; 85: 352-361.

25 Brander P E, Mortimore I L, Douglas N J. Effect of obesity and erect/supine posture on lateral cephalometry: relationship to sleepdisordered breathing. Eur Resp J 1999; 13: 398-402.

26 Friedlander A H, Yueh R, Littner M R. The prevalence of calcified carotid artery atheromas in patients with obstructive sleep apnoea. J Oral Maxillofac Surg 1998; 56: 950-954.

27 Jenkinson C, Davies R J O, Mullins R, Stradling J R. Comparison of therapeutic and subtherapeutic nasal continuous positive airway pressure for obstructive sleep apnoea: a randomized prospective parallel trial. Lancet 1999; 353: 2100-2105.

28 Strobel R J, Rosen R C. Obesity and weight loss in obstructive sleep apnoea: a critical review. Sleep 1996; 19: 104-115.

29 Engleman H M, Martin S E, Deary I J, Douglas $\mathrm{N} \mathrm{J}$. Effect of continuous positive airway pressure treatment on daytime function in sleep apnoea/hypopnoea syndrome. Lancet 1994; 343: 572-575.

30 Polo O. Continuous positive airway pressure for treatment of sleep apnoea. Lancet 1999; 353: 2086-2087.

31 Stradling J. Sleep apnoea and the misuse of evidence-based medicine. Lancet 1997; 349: 201-202.

32 Jenkinson C. The SF-36 physical and mental health summary measures: an example of how to interpret scores. J Health Serv Res Policy 1998; 3: 92-96.

33 The Australian Health Technology Advisory Committee. The effectiveness and costeffectiveness of nasal continuous positive airway pressure ( $n C P A P)$ in the treatment of obstructive sleep apnea in adults. Canberra: Government Public Service, 1996.

34 Tousignant P, Cosio M G, Levy R D, Groome P A. Quality adjusted life years added by treatment of obstructive sleep apnoea. Sleep 1994; 17: 52-60.

35 Wessex Institute of Public Health Medicine. Continuous positive airway pressure for obstructive sleep apnoea. Health Tech Evaluation Rev,1994, publication No. 31.

36 Polo O, Berthon-Jones M, Douglas N J, Sullivan C E. Management of obstructive sleep apnoea/hypopnoea syndrome. Lancet 1994; 344: 656-660.

37 Teschler H, Berthon-Jones M. Intelligent CPAP systems: clinical experience. Thorax 1998; 53(Suppl 3): S49-54

38 Gibson G J, Douglas N J, Stradling J R, London D R, Semple S J G. Sleep apnoea: clinical importance and facilities for investigation and treatment in the UK. Addendum to the 1993 Royal College of Physicians Sleep Apnoea report. J Royal Coll Physicians 1998; 32: 540-544.

39 Ferguson K A, Ono T, Lowe A A, Keenan S P, Fleetham J A. A cross-over study of an oral appliance vs. nasal continuous positive airway pressure in the treatment of mild-moderate obstructive sleep apnoea. Chest 1996; 109: 1269-1275.
40 Schmidt-Nowara W W, Meade T E, Hays M B. Treatment of snoring and obstructive sleep apnea with a dental orthosis. Chest 1991; 99. 1378-1385.

41 O'Sulivan R A, Hillman D R, Mateljan R, Pantin C, Finucane K E. Mandibular advancement splint: an appliance to treat snoring and obstructive sleep apnea. Am J Respir Crit Care Med 1995; 151: 194-198.

42 Eveloff S E, Rosenberg C L. Carlisle C C, Millman R P. Efficacy of a Herbst mandibular advancement device in obstructive sleep apnea. Am J Respir Crit Care Med 1994; 149: 905-909.

43 Cohen R. Obstructive sleep apnoea: oral appliance therapy and severity of condition. Oral Surg Oral Med Oral Path Oral Radiol Endo 1998; 85: 388-392.

44 Hans M G, Nelson S, Luks V G, Lorkovich P, Baek S. Comparison of two dental devices for treatment of obstructive sleep apnoea (OSAS). Am J Orthod Dentofac Orthoped 1997; 111: 562570.

45 Bennett L S, Davies R J, Stradling J R. Oral appliances for the management of snoring and obstructive sleep apnoea. Thorax 1998; 53(Suppl 2): S58-64

46 Eckhart JE. Comparisons of oral devices for snoring. J Cal Dent Assoc 1998; 26: 611-623.

47 Ferguson K A, Ono T, Lowe A A, Al-Majed S, Love L, Fleetham J A. A short term controlled trial of an adjustable oral appliance for the treatment of mild to moderate obstructive sleep apnoea. Thorax 1997; 52: 362-368.

48 Stradling J R, Negus T W, Smith D, Langford B. Mandibular advancement devices for control of snoring. Eur Resp J 1998; 11: 447-450.

49 Carney A S, Robinson P J. Assessment and management of snoring: a surgical perspective. BJ Hosp Med 1995; 53: 515-512.

50 Battagel J M. Obstructive sleep apnea: fact not fiction. Br J Orthod 1996; 23: 315-324.

51 Waite P D, Wooten V, Lachner J, Guyett R F. Maxillomandibular advancement surgery in 23 patients with obstructive sleep apnoea. J Oral Maxillofac Surg 1989; 47: 1256-1261.

52 Wilfang J, Merten H A, Luhr H G. The functional palatorrhapy in the treatment of obstructive sleep apnoea. Br J Oral Maxillofac Surg 1996; 34: 82-86.

53 Powell N B, Riley R W, Guilleminault C. Maxillofacial surgical techniques for hypopharyngeal obstruction in obstructive sleep apnea. Oper Tech Otolaryngol Head Neck Surg 1991; 2: 112-119.

54 Riley R W, Powell N B, Guilleminault C. Obstructive sleep apnea syndrome: a review of 306 consecutively treated surgical patients. Otolaryngol Head Neck Surg 1993; 108: 117-125.

55 Waite P D, Shttar S M. Maxillomandibular advancement surgery: a cure for obstructive sleep apnea syndrome. Oral Maxillofac Surg Clin N Am 1995; 7: 327-336.

56 Fairbanks D N F. Tracheostomy for obstructive sleep apnea: Indications and technique. In: Fairbanks DNF, Fujita S (eds) Snoring and Obstructive Sleep Apnea. New York: Raven Press, 1994, pp. 169-177. 